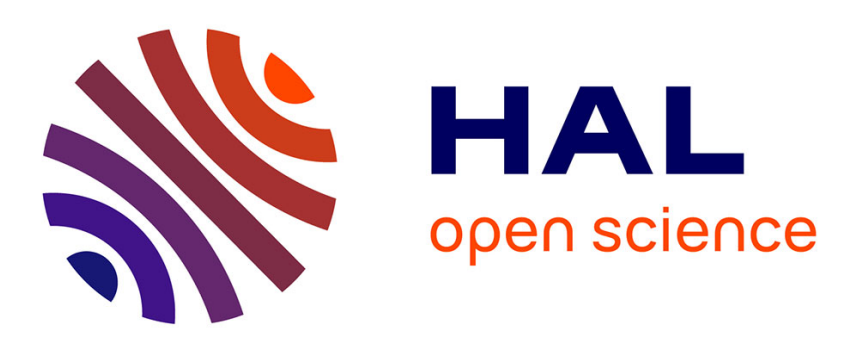

\title{
Unified Theoretical and Experimental View on Transient Shear Banding
}

Roberto Benzi, Thibaut Divoux, Catherine Barentin, Sébastien Manneville, Mauro Sbragaglia, Federico Toschi

\section{> To cite this version:}

Roberto Benzi, Thibaut Divoux, Catherine Barentin, Sébastien Manneville, Mauro Sbragaglia, et al.. Unified Theoretical and Experimental View on Transient Shear Banding. Physical Review Letters, 2019, 123 (24), pp.248001. 10.1103/PhysRevLett.123.248001 . hal-02406078

\section{HAL Id: hal-02406078 https://hal.science/hal-02406078}

Submitted on 11 Feb 2021

HAL is a multi-disciplinary open access archive for the deposit and dissemination of scientific research documents, whether they are published or not. The documents may come from teaching and research institutions in France or abroad, or from public or private research centers.
L'archive ouverte pluridisciplinaire HAL, est destinée au dépôt et à la diffusion de documents scientifiques de niveau recherche, publiés ou non, émanant des établissements d'enseignement et de recherche français ou étrangers, des laboratoires publics ou privés. 


\title{
Unified Theoretical and Experimental View on Transient Shear Banding
}

\author{
Roberto Benzi, ${ }^{1}$ Thibaut Divoux $\odot,{ }^{2,3}$ Catherine Barentin, ${ }^{4}$ Sébastien Manneville, ${ }^{2,5}$ \\ Mauro Sbragaglia, ${ }^{1}$ and Federico Toschi ${ }^{6}$ \\ ${ }^{1}$ Dipartimento di Fisica, Università di Roma "Tor Vergata" and INFN, Via della Ricerca Scientifica, 1-00133 Roma, Italy \\ ${ }^{2}$ MultiScale Material Science for Energy and Environment, UMI 3466, CNRS-MIT, \\ 77 Massachusetts Avenue, Cambridge, Massachusetts 02139, USA \\ ${ }^{3}$ Department of Civil and Environmental Engineering, Massachusetts Institute of Technology, Cambridge, Massachusetts 02139, USA \\ ${ }^{4}$ Université de Lyon, Université Claude Bernard Lyon 1, CNRS, Institut Lumière Matière, F-69622 Villeurbanne, France \\ ${ }^{5}$ Univ Lyon, Ens de Lyon, Univ Claude Bernard, CNRS, Laboratoire de Physique, F-69342 Lyon, France \\ ${ }^{6}$ Department of Applied Physics, Eindhoven University of Technology, P.O. Box 513, 5600 MB Eindhoven, Netherlands
}

(Received 20 July 2019; published 9 December 2019)

\begin{abstract}
Dense emulsions, colloidal gels, microgels, and foams all display a solidlike behavior at rest characterized by a yield stress, above which the material flows like a liquid. Such a fluidization transition often consists of long-lasting transient flows that involve shear-banded velocity profiles. The characteristic time for full fluidization $\tau_{f}$ has been reported to decay as a power law of the shear rate $\dot{\gamma}$ and of the shear stress $\sigma$ with respective exponents $\alpha$ and $\beta$. Strikingly, the ratio of these exponents was empirically observed to coincide with the exponent of the Herschel-Bulkley law that describes the steady-state flow behavior of these complex fluids. Here we introduce a continuum model, based on the minimization of a "free energy," that captures quantitatively all the salient features associated with such transient shear banding. More generally, our results provide a unified theoretical framework for describing the yielding transition and the steady-state flow properties of yield stress fluids.
\end{abstract}

DOI: $10.1103 /$ PhysRevLett.123.248001

Introduction.-Amorphous soft materials, such as dense emulsions, foams, and microgels, display solidlike properties at rest, while they flow like liquids for large enough stresses [1-4]. These yield stress fluids are characterized by a steady-state flow behavior that is well described by the Herschel-Bulkley (HB) model, where the shear stress $\sigma$ is linked to the shear rate $\dot{\gamma}$ through $\sigma=\sigma_{c}+A \dot{\gamma}^{n}$, with $\sigma_{c}$ the yield stress of the fluid, $A$ the consistency index, and $n$ a phenomenological exponent that ranges between 0.3 and 0.7 , and is often equal to $1 / 2$ [5-8]. However, steady-state flow is never reached instantly and the yielding transition may involve transient regimes much longer than the natural timescale $\dot{\gamma}^{-1}$ [4,9-13].

As demonstrated experimentally in Refs. [14-16], longlasting heterogeneous flows develop from the initial solidlike state, involving shear-banded velocity profiles before reaching a homogeneous steady-state flow. Depending on the imposed variable, $\dot{\gamma}$ or $\sigma$, the characteristic time $\tau_{f}$ to reach a fully fluidized state was reported to scale, respectively, as $\tau_{f} \propto 1 / \dot{\gamma}^{\alpha}$ or as $\tau_{f} \propto 1 /\left(\sigma-\sigma_{c}\right)^{\beta}$, where $\alpha$ and $\beta$ are fluidization exponents that only depend on the material properties (see Fig. 1). Interestingly, these two power laws naturally lead to a constitutive relation $\sigma$ versus $\dot{\gamma}$ given by the steady-state HB equation with an exponent $n=\alpha / \beta[15]$.

The above experimental findings have triggered a wealth of theoretical contributions aiming at reproducing long-lasting heterogeneous flows, some of which have successfully produced transient shear-banded flows together with nontrivial scaling laws for fluidization times [18-24]. While these contributions offer potential explanations for

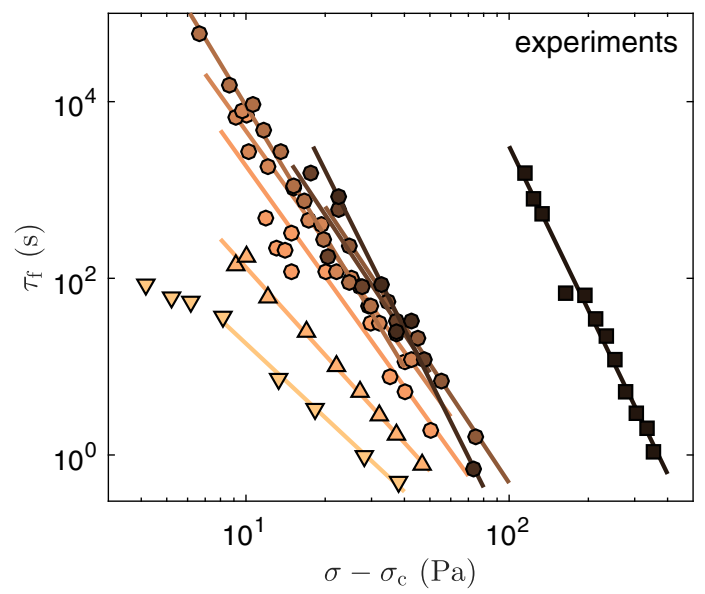

FIG. 1. Stress-induced fluidization time $\tau_{f}$ versus reduced shear stress $\sigma-\sigma_{c}$ for Carbopol microgels at various weight concentrations: $0.5 \%$ (up-pointing triangle), $0.7 \%$ (down-pointing triangle), $1 \%$ (circle), and 3\% (rectangle). Solid lines correspond to the best power-law fits of the various data sets $\tau_{f} \sim\left(\sigma-\sigma_{c}\right)^{-\beta}$ with exponent $\beta$ ranging from 2.8 to 6.2. Experimental conditions are listed in Supplemental Material Table S1 together with values of $\sigma_{c}$ and $\beta$ [17]. 
long-lasting transients, which appear to be age dependent and related to structural heterogeneities [19,21-23,25], none of these numerical studies captures the link between the exponents governing the transient regimes and that of the steady-state HB behavior.

From a more general perspective, shear banding has often been discussed as a first-order dynamical phase transition [13,26-29]. In that framework, transient shear banding can be interpreted as the coarsening of the fluid phase, which nucleates within the solid region and whose size $\delta$ can be seen as the growing length scale that characterizes the coarsening dynamics. In this Letter, we show that the yielding transition and the corresponding transient shear-banding behavior can be described by a field theory based on a "free energy," whose order parameter is the fluidity, i.e., the ratio between the shear rate and the shear stress. In such a theory, as first introduced by Bocquet et al. [28] and later analyzed in Ref. [30], shearbanded flows can be obtained as a minimum of a free energy that depends on the fluidity and on the nonlocal, i.e., spatially dependent [26,27], rheological properties of the system. A link between the fluidity order parameter and the physics of elastoplasticity at the mesoscale has been explored in Ref. [31] based on Eshelby elastic response functions [32-34]. Here we build upon the fluidity approach and extend it, leading to analytical expressions for the scaling exponents $\alpha$ and $\beta$ that are in quantitative agreement with experiments and that provide a clear-cut explanation for the link between these exponents and the HB exponent $n$. Our findings demonstrate that nonlocal effects are key to understand transient shear banding in amorphous soft solids.

Fluidity model.-We start by considering that the bulk rheology of the system is governed by the dimensionless HB model, $\Sigma=1+\dot{\Gamma}^{n}$, where $\Sigma=\sigma / \sigma_{c}$ is the shear stress normalized by the yield stress and $\dot{\Gamma}=\dot{\gamma} /\left(\sigma_{c} / A\right)^{1 / n}$ is the shear rate normalized by the characteristic frequency for the HB law. Given the spatial coordinate $y$ along the velocity gradient direction and the system size $L$, we next assume that the flow properties of the yield stress fluid are controlled by a free-energy functional, $F[f]=\int_{0}^{L} \Phi(f, m, \xi) d y$, where $[28,35]$

$$
\Phi(f, m, \xi) \equiv\left[\frac{1}{2} \xi^{2}(\nabla f)^{2}-\frac{1}{2} m f^{2}+\frac{2}{5} f^{5 / 2}\right] .
$$

The quantity $f=f(y)$ is the local (dimensionless) fluidity defined by $f(y)=\dot{\Gamma}(y) / \Sigma(y)$ and represents the order parameter in the model. Following Refs. [28,30], $\mathrm{m}^{2}$ is defined as

$$
m^{2}(\Sigma) \equiv \frac{(\Sigma-1)^{1 / n}}{\Sigma} \quad \text { for } \Sigma \geq 1
$$

and $m^{2}=0$ for $\Sigma<1$. This formulation implies that, for $f(y)=m^{2}$ independently of $y$, the system flows homogeneously and follows the dimensionless HB model.
Finally, the length scale $\xi$ is usually referred to as the "cooperative" scale and is of the order of a few times the size of the elementary microstructural constituents [28,36-39]. In steady state, the flowing properties of the system can then be derived from the variational equation $\delta F / \delta f=0$. This equation predicts heterogeneous flow profiles as induced by wall effects, but it cannot account for stable shear banding [30]. Moreover, transient flow properties require that some temporal dynamics be specified for $f$. To overcome these limitations, we now generalize a recent theoretical proposal introduced in Ref. [30] and apply it to describe transient flows.

Stress-induced fluidization dynamics.-Let us first focus on the yielding transition under an imposed shear stress $\sigma$ for which $m$ is a constant. We note that introducing $\tilde{f}=f / m^{2}$ and $\tilde{y}=m^{1 / 2} y / \xi$ allows us to rescale homogeneously the functional $\Phi$ to $\Phi(f, m, \xi)=m^{5} \tilde{\Phi}(\tilde{f})$, where [40]

$$
\tilde{\Phi}(\tilde{f})=\left[\frac{1}{2}(\tilde{\nabla} \tilde{f})^{2}-\frac{1}{2} \tilde{f}^{2}+\frac{2}{5} \tilde{f}^{5 / 2}\right] .
$$

The advantage of using $\tilde{f}$ and $\tilde{y}$ is that we can now formulate the dynamical equation independently of both the strength of external forcing $m$ and $\xi$. We further assume that the system reaches a stable equilibrium configuration corresponding to a minimum of $F[\tilde{f}]$ and that such dynamics is governed by a "mobility" $k(\tilde{f})$, for which the most general dynamical equation reads [35]

$$
\begin{aligned}
\frac{\partial \tilde{f}}{\partial t} & =-m^{5} k(\tilde{f}) \frac{\delta F[\tilde{f}]}{\delta \tilde{f}} \\
& =m^{5} k(\tilde{f})\left[\tilde{\Delta} \tilde{f}+\tilde{f}-\tilde{f}^{3 / 2}\right] .
\end{aligned}
$$

If the mobility $k(\tilde{f})$ is an analytic function of $\tilde{f}$ and $k(0)=0$, then Eq. (4) can account for a shear-banding solution in the general form $\tilde{f}(\tilde{y})=0$ (solid branch) for $\tilde{y} \in[0, \tilde{L}-\tilde{\delta}]$ and $\tilde{f}(\tilde{y})$ solution of $\tilde{\Delta} \tilde{f}+\tilde{f}-\tilde{f}^{3 / 2}=0$ (fluidized branch) for $\tilde{y} \in[\tilde{L}-\tilde{\delta}, \tilde{L}]$, where $\tilde{\delta}$ is the rescaled size of the fluidized region. Furthermore, transient shear banding corresponds to the case where the solid branch $\tilde{f}=0$ is an unstable solution. To explore this latter case, we next consider the time dynamics in Eq. (4) with $k(\tilde{f})=\tilde{f}$ and fixed initial conditions. Note that the initial conditions influence mainly the early-time response of the fluid. A detailed discussion on the choice of $k(\tilde{f})$ and on initial conditions is given in the Supplemental Material [17]. Equation (4) is solved numerically for $\Sigma=1.1$ and $\xi / L=$ 0.01 in Figs. 2(a) and 2(b), assuming $\tilde{f}(\tilde{y}, 0)=\tilde{f}_{0} \ll 1$ for the initial solidlike state and $\tilde{f}(\tilde{L}, t)=1$ and $\partial_{\tilde{y}} \tilde{f}(0, t)=0$ for boundary conditions at the two different walls. Such a choice will be addressed below in the discussion section. As seen in the velocity profiles $v(y)$ [insets in Fig. 2(a)], the 

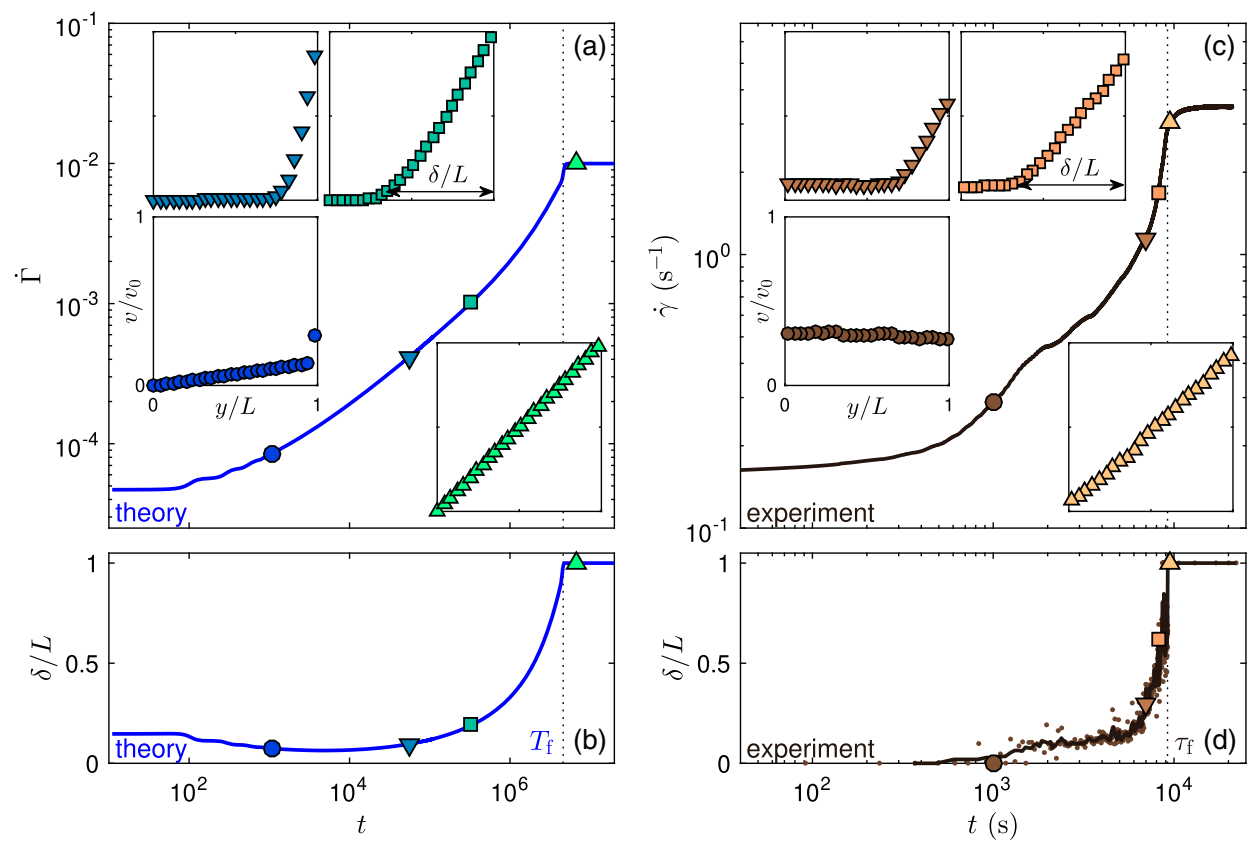

FIG. 2. Stress-induced fluidization dynamics in (a),(b) theory and (c),(d) experiment on a $1 \mathrm{wt} \%$ Carbopol microgel in a smooth concentric cylinder geometry of gap $1 \mathrm{~mm}$. (a),(c) Shear rate $\dot{\Gamma}$ and $\dot{\gamma}$ versus time $t$ for a shear stress of $\Sigma=1.1$ and $\sigma=41 \mathrm{~Pa}$, respectively. Insets: Velocity profiles $v$ normalized by the velocity of the moving plate $v_{0}$ as a function of the distance $y$ to the fixed plate normalized by the gap size $L$. Profiles taken at different times (symbol, time): (circle, 1100), (down-pointing triangle, $5.5 \times 10^{4}$ ), (square, $3.3 \times 10^{5}$ ), (up-pointing triangle, $6.6 \times 10^{6}$ ) in (a) and (circle, $1011 \mathrm{~s}$ ), (down-pointing triangle, $6927 \mathrm{~s}$ ), (square, $8193 \mathrm{~s}$ ); (up-pointing triangle, $9522 \mathrm{~s}$ ) in (c). (b),(d) Width $\delta$ of the fluidized shear band normalized by the gap width $L$ versus time $t$. The vertical dashed lines crossing (a),(b) and (c),(d), respectively, indicate the fluidization times $T_{f}$ and $\tau_{f}$.

system forms a shear band near $y=L$ at time $t>0$. The shear band grows in time and the system eventually reaches the stable equilibrium configuration $\tilde{f}(\tilde{y}, t)=1$ within a well-defined fluidization time $T_{f}$. This phenomenology is in remarkable agreement with experimental observations in Figs. 2(c) and 2(d) for a Carbopol microgel. In particular, the band size $\delta(t)$ follows very similar growths whatever the applied stress (see Fig. S1 in Supplemental Material [17]).

Using Eq. (4), we may predict the scaling behavior of the fluidization time $T_{f}$ as a function of $m$. Upon rescaling the time as $\tilde{t}=m^{5} t$, we observe that Eq. (4) no longer depends on $m$. Regardless of the specific function $k(\tilde{f})$, we expect that the shear band expands with some characteristic velocity $\tilde{v}_{f}$ independent of $m$. Therefore, the rescaled fluidization time should be proportional to $\tilde{L} / \tilde{v}_{f}$. It follows that the fluidization time should exhibit the scaling $T_{f} \sim$ $\tilde{L} /\left(m^{5} \tilde{v}_{f}\right) \sim 1 /\left(\xi m^{9 / 2}\right)$ independently of the specific functional form of $k(\tilde{f})$. The numerical integration of Eq. (4) for various values of $m$ leads to the fluidization times $T_{f}$ shown in Fig. 3(a), which nicely follow the predicted $m^{-9 / 2}$ power-law decay. Such a scaling is also in excellent agreement with the experimental data of Fig. 1 when rescaled and plotted in terms of $m(\Sigma)$ based on the experimental steady-state HB parameters [see Fig. 3(b) and discussion below].
Strain-induced fluidization.-We now proceed to show that the same approach allows us to rationalize the yielding transition under an imposed shear rate $\dot{\Gamma}$. In that case, we must supplement the theory by the fluidity equation $\dot{\Sigma}=\dot{\Gamma}-f \Sigma$, which corresponds to a single Maxwell mode for the evolution of the stress [19]. Moreover, $m$ being a function of time, we can no longer use the rescaling $\tilde{f}=f / m^{2}$. Since $\dot{\Gamma}$ is a constant, we rather introduce the rescaled variable $\tilde{f}=f / \dot{\Gamma}$. Upon rescaling the spatial variable as $\tilde{y}=\dot{\Gamma}^{1 / 4} y / \xi$, the analog of Eq. (4) reads

$$
\frac{\partial \tilde{f}}{\partial t}=\dot{\Gamma}^{5 / 2} k(\tilde{f})\left[\tilde{\Delta} \tilde{f}+\tilde{m} \tilde{f}-\tilde{f}^{3 / 2}\right],
$$

where $\tilde{m}=m / \dot{\Gamma}^{1 / 2}$. Under the assumption that $\tilde{m}$ remains roughly constant during the shear band evolution, rescaling time as $\tilde{t}=\dot{\Gamma}^{5 / 2} t$ leads to $T_{f} \sim \tilde{L} /\left(\dot{\Gamma}^{5 / 2} \tilde{v}_{f}\right) \sim 1 /\left(\xi \dot{\Gamma}^{9 / 4}\right)$. The inset of Fig. 4 shows the actual $T_{f}$ computed numerically from Eq. (5) with $k(\tilde{f})=\tilde{f}$ for different shear rates $\dot{\Gamma}$. The results are very well fitted by a power-law decay of exponent $2.15 \pm 0.10$, quite close to the theoretical exponent $\alpha=9 / 4$, and in good agreement with experiments on a $1 \mathrm{wt} \%$ Carbopol microgel for various geometries and boundary conditions that lead to an exponent of $2.45 \pm 0.23$ (see Fig. 4 herein and Table S2 in Supplemental Material [17]). 


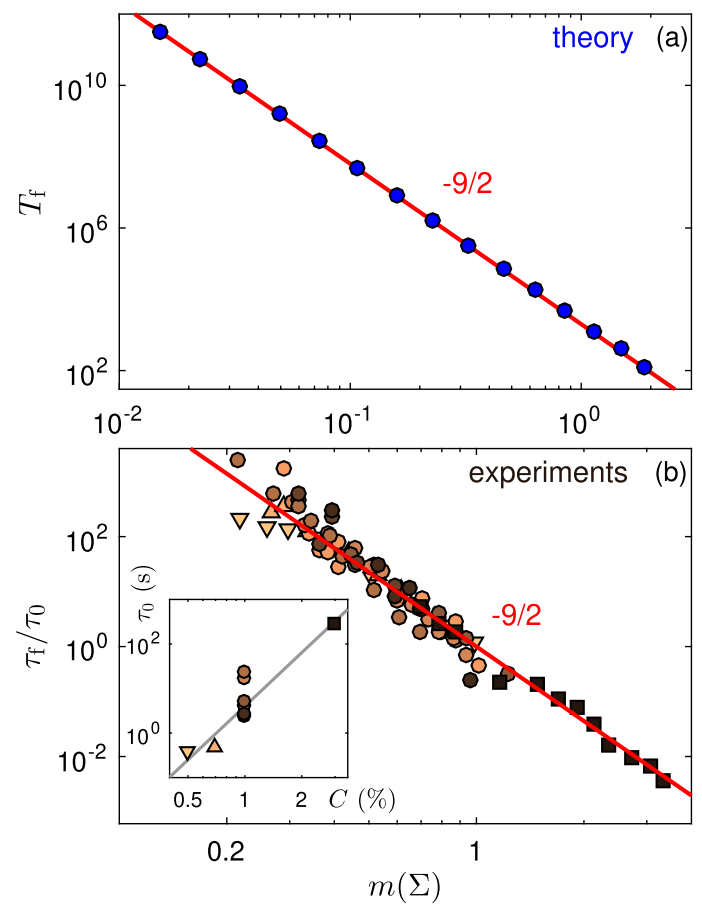

FIG. 3. Stress-induced fluidization time as a function of $m(\Sigma)$ defined by Eq. (2). (a) Theoretical predictions $T_{f}$. (b) Experiments from Fig. 1 where each dataset for $\tau_{f}$ was rescaled by the time $\tau_{0}$ shown in the inset as a function of the microgel concentration $C$ (see also Supplemental Material Table S1 [17]). Red lines show the predicted power law with exponent $-9 / 2$. The best power-law fits of the whole datasets yield exponents $-4.46 \pm 0.10$ and $-4.69 \pm 0.33$, respectively, for theory and experiments. The gray line in the inset is $\tau_{0} \sim C^{4}$.

Discussion.-Let us now compare the theoretical findings against experimental data. Coming back to the case of an imposed shear stress and to the definition of $m$ in Eq. (2), we note that $T_{f} \sim m^{-9 / 2}$ corresponds to the scaling $T_{f} \sim$ $(\Sigma-1)^{-9 / 4 n}$ in terms of the reduced viscous stress $\Sigma-1$. This corresponds to a fluidization exponent $\beta=9 / 4 n$. To illustrate such a scaling, numerical results are plotted in Fig. S2 of Supplemental Material for different values of $n$ covering the range reported in experiments $(n \simeq 0.30-0.57)$ [17]. The spread of the exponents $\beta \simeq 3-8$ nicely corresponds to that observed experimentally $(\beta \simeq 2.8-6.2)$. More specifically, these theoretical predictions prompt us to revisit the experimental data shown in Fig. 1 by computing estimates of $m(\Sigma)$ using Eq. (2) with $\Sigma=$ $\sigma / \sigma_{c}$ and the HB parameters $\sigma_{c}$ and $n$ determined at steady state [15]. When plotted as a function of $m(\Sigma)$, the experimental fluidization times remarkably collapse onto the predicted scaling $\tau_{f} \sim m(\Sigma)^{-9 / 4}$, provided $\tau_{f}$ is rescaled by a characteristic time $\tau_{0}$ independent of the applied stress [see Fig. 3(b)]. Although a clear physical interpretation of $\tau_{0}$ is still lacking [41], the collapse of the experimental data seen in Fig. 3(b) is a strong signature of the predictive power of the theory.

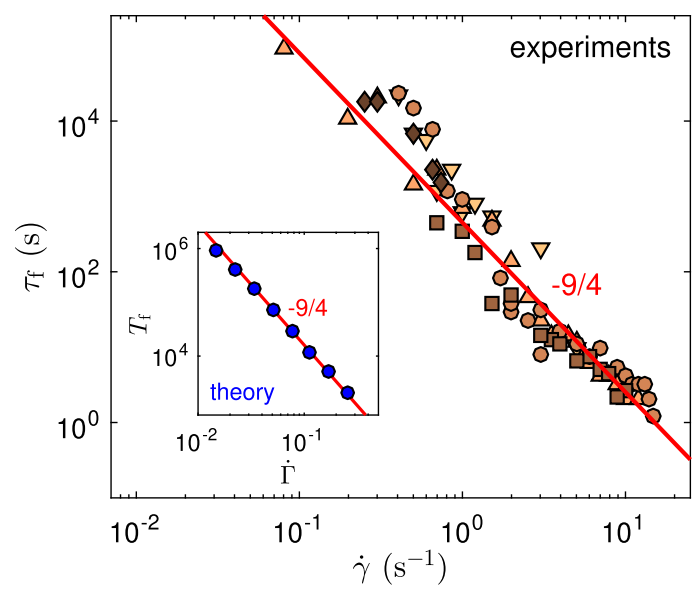

FIG. 4. Strain-induced fluidization time $\tau_{f}$ versus shear rate $\dot{\gamma}$ for a $1 \mathrm{wt} \%$ Carbopol microgel under the various experimental conditions listed in Supplemental Material Table S2 [17]. Inset: Theoretical prediction for $T_{f}$ versus $\dot{\Gamma}$. Red lines show the predicted power law with exponent $-9 / 4$. The best power-law fits of the whole datasets yield exponents $-2.15 \pm 0.10$ and $-2.45 \pm 0.23$, respectively, for theory and experiments.

Another key outcome of the proposed approach is that, assuming an underlying $\mathrm{HB}$ rheology, it provides the first theoretical analytical expressions for both fluidization exponents $\alpha$ and $\beta$, in quantitative agreement with experimental results. Moreover, the ratio of these exponents, $\alpha / \beta=(9 / 4) /(9 / 4 n)=n$, coincides with the HerschelBulkley exponent exactly as in experiments $[15,16]$. Therefore, the present theory provides a natural framework for justifying the empirical connection between transient and steady-state flow behaviors.

Furthermore, the scaling found here for $\tau_{f}$ is extremely robust and depends only weakly on the initial conditions. As illustrated in Figs. S3 and S4 of Supplemental Material for two different initial values of the fluidity in the gap, the shear rate either shows a monotonic increase up to complete fluidization or displays a decreasing trend with a welldefined minimum before increasing towards steady state [17]. Yet, the fluidization time remains comparable in both cases. Note also that, at early stage, $\dot{\Gamma}$ shows a power-law decrease in time that is strongly reminiscent of the primary creep regime reported in amorphous soft materials $[11,15,42-46]$. In the present model, the power-law exponent may take any value between $-2 / 3$ and 0 depending on the choice of $k(\tilde{f})$, thus providing an explanation for the diversity of exponents reported in the literature.

To conclude, our results show that the "free-energy" approach originally introduced to account for nonlocal effects in steady-state flows of complex fluids [28] also captures long-lasting transient heterogeneous flows: thanks to cooperative effects, a fluidized band nucleates and grows until complete yielding, which quantitatively matches the experimental phenomenology. In this framework, transient shear banding appears as the dynamical signature of the 
unstable nature of the solid branch at $\dot{\gamma}=0$ in the flow curve $[4,47,48]$. More generally, as explored in Ref. [30], the present model also accounts for steady-state shear banding when cooperative effects are hindered, e.g., by mechanical noise that prevents the shear band from growing through cascading plastic events. Such a connection between transient and steady-state behaviors in terms of cooperativity-induced stability of the shear band offers for the first time a unified framework for describing the local scenario associated with the yielding dynamics of soft glassy materials.

The authors thank David Tamarii for help with the experiments as well as Emanuela Del Gado and Suzanne Fielding for fruitful discussions. This research was supported in part by the National Science Foundation under Grant No. NSF PHY-1748958 through the KITP program on the Physics of Dense Suspensions.

[1] H. A. Barnes, J. Non-Newtonian Fluid Mech. 81, 133 (1999).

[2] N. Balmforth, I. Frigaard, and G. Ovarlez, Annu. Rev. Fluid Mech. 46, 121 (2014).

[3] P. Coussot, J. Non-Newtonian Fluid Mech. 211, 31 (2014).

[4] D. Bonn, M. M. Denn, L. Berthier, T. Divoux, and S. Manneville, Rev. Mod. Phys. 89, 035005 (2017).

[5] W. H. Herschel and R. Bulkley, Kolloid Z. 39, 291 (1926).

[6] H. Barnes and Q. Nguyen, J. Non-Newtonian Fluid Mech. 98, 1 (2001).

[7] G. Katgert, M. E. Möbius, and M. van Hecke, Phys. Rev. Lett. 101, 058301 (2008).

[8] S. Cohen-Addad and R. Höhler, Curr. Opin. Colloid Interface Sci. 19, 536 (2014).

[9] J. Sprakel, S. B. Lindström, T. E. Kodger, and D. A. Weitz, Phys. Rev. Lett. 106, 248303 (2011).

[10] M. Siebenbürger, M. Ballauff, and T. Voigtmann, Phys. Rev. Lett. 108, 255701 (2012).

[11] V. Grenard, T. Divoux, N. Taberlet, and S. Manneville, Soft Matter 10, 1555 (2014).

[12] S. Fielding, Rep. Prog. Phys. 77, 102601 (2014).

[13] T. Divoux, M.-A. Fardin, S. Manneville, and S. Lerouge, Annu. Rev. Fluid Mech. 48, 81 (2016).

[14] T. Divoux, D. Tamarii, C. Barentin, and S. Manneville, Phys. Rev. Lett. 104, 208301 (2010).

[15] T. Divoux, C. Barentin, and S. Manneville, Soft Matter 7, 8409 (2011).

[16] T. Divoux, D. Tamarii, C. Barentin, S. Teitel, and S. Manneville, Soft Matter 8, 4151 (2012).

[17] See Supplemental Material at http://link.aps.org/ supplemental/10.1103/PhysRevLett.123.248001 for more details on experiments and theoretical considerations.

[18] X. Illa, A. Puisto, A. Lehtinen, M. Mohtaschemi, and M. J. Alava, Phys. Rev. E 87, 022307 (2013).

[19] R. L. Moorcroft, M. E. Cates, and S. M. Fielding, Phys. Rev. Lett. 106, 055502 (2011).

[20] R. L. Moorcroft and S. M. Fielding, Phys. Rev. Lett. 110, 086001 (2013).
[21] A. R. Hinkle and M. L. Falk, J. Rheol. 60, 873 (2016).

[22] V. V. Vasisht, G. Roberts, and E. del Gado, arXiv: $1709.08717 \mathrm{v} 1$.

[23] C. Liu, K. Martens, and J.-L. Barrat, Phys. Rev. Lett. 120, 028004 (2018).

[24] A. Jain, R. Singh, L. Kushwaha, V. Shankar, and Y. M. Joshi, J. Rheol. 62, 1001 (2018).

[25] C. Liu, E. E. Ferrero, K. Martens, and J.-L. Barrat, Soft Matter 14, 8306 (2018).

[26] J. K. G. Dhont, Phys. Rev. E 60, 4534 (1999).

[27] C.-Y. David Lu, P. D. Olmsted, and R. C. Ball, Phys. Rev. Lett. 84, 642 (2000).

[28] L. Bocquet, A. Colin, and A. Ajdari, Phys. Rev. Lett. 103, 036001 (2009).

[29] V. Chikkadi, D. M. Miedema, M. T. Dang, B. Nienhuis, and P. Schall, Phys. Rev. Lett. 113, 208301 (2014).

[30] R. Benzi, M. Sbragaglia, M. Bernaschi, S. Succi, and F. Toschi, Soft Matter 12, 514 (2016).

[31] A. Nicolas and J.-L. Barrat, Phys. Rev. Lett. 110, 138304 (2013)

[32] J. D. Eshelby, Proc. R. Soc. A 241, 376 (1957).

[33] V. Hieronymus-Schmidt, H. Rösner, G. Wilde, and A. Zaccone, Phys. Rev. B 95, 134111 (2017).

[34] R. Dasgupta, H. G. Hentschel, and I. Procaccia, Phys. Rev. Lett. 109, 255502 (2012).

[35] For simplicity, we shall use the same symbols $y$ and $t$ for the spatial and temporal degrees of freedom in both experiments and theory.

[36] J. Goyon, A. Colin, G. Ovarlez, A. Ajdari, and L. Bocquet, Nature (London) 454, 84 (2008).

[37] J. Goyon, A. Colin, and L. Bocquet, Soft Matter 6, 2668 (2010).

[38] B. Géraud, L. Bocquet, and C. Barentin, Eur. Phys. J. E 36, 30 (2013).

[39] B. Géraud, L. Jorgensen, C. Ybert, H. Delanoë-Ayari, and C. Barentin, Eur. Phys. J. E 40, 5 (2017).

[40] The tildes over the gradient $\nabla$ and Laplacian $\Delta$ operators in Eqs. (3)-(5) indicate that derivatives are taken over the rescaled spatial variable $\tilde{y}$.

[41] The rescaling factor $\tau_{0}$ strongly depends on the system concentration $C$, scaling roughly as $C^{4}$, as shown in the inset of Fig. 3(b), and does not appear to be trivially linked to the characteristic time $\left(A / \sigma_{c}\right)^{1 / n}$ that one can extract from the $\mathrm{HB}$ behavior, which only varies from 0.12 to $0.61 \mathrm{~s}$ in our experiments.

[42] T. Bauer, J. Oberdisse, and L. Ramos, Phys. Rev. Lett. 97, 258303 (2006).

[43] M. Leocmach, C. Perge, T. Divoux, and S. Manneville, Phys. Rev. Lett. 113, 038303 (2014).

[44] A. Helal, T. Divoux, and G. H. McKinley, Phys. Rev. Applied 6, 064004 (2016).

[45] P. Lidon, L. Villa, and S. Manneville, Rheol. Acta 56, 307 (2017).

[46] S. Aime, L. Ramos, and L. Cipelletti, Proc. Natl. Acad. Sci. U.S.A. 115, 3587 (2018).

[47] F. Varnik, L. Bocquet, J.-L. Barrat, and L. Berthier, Phys. Rev. Lett. 90, 095702 (2003).

[48] F. Varnik, L. Bocquet, and J.-L. Barrat, J. Chem. Phys. 120, 2788 (2004). 